\title{
SELECCIÓN DE LEGUMINOSAS DE HÁBITO TREPADOR TIPO IV EN ASOCIO CON MAÍZ PARA ALIMENTACIÓN BOVINA
}

\author{
Dilmer Gabriel Guerra-Guzmán ${ }^{1}$, Jorge Ernesto Guevara-Ohara ${ }^{2 / *}$, \\ Henris Jobany Morazán-Nuñez ${ }^{3}$
}

\begin{abstract}
Palabras clave: Calidad forrajera; proteína; sistemas de cultivo; índice de selección ponderada (ISP); índice de equivalencia de área (IEA).

Keywords: Forage quality; protein; farming systems; weighted selection index (WSI); area equivalence index (AEI).
\end{abstract}

Recibido: $11 / 05 / 2020$

RESUMEN

Introducción. La producción de rumiantes depende casi exclusivamente de forrajes que deseablemente ostenten una calidad nutritiva alta, por lo que estudios actualizados sobre ese atributo, son requeridos. Para el presente estudio, se realizó un análisis del asocio de gramíneas y leguminosas como alternativas para incrementar el valor nutricional de los forrajes. Objetivo. Valorar el comportamiento agronómico y bromatológico de asocios de maíz con leguminosas de hábito trepador IV para la alimentación bovina. Materiales y métodos. Se utilizó un diseño de bloques completos al azar con 7 tratamientos y 3 repeticiones conformados por: $\mathrm{T}_{1-6}=$ Maíz+ Lago azul, Beijín, Chi tou, Pritkar, Judías, CIAT 16806 y $\mathrm{T}_{7}=$ Maíz ICA-V305. Se evaluó la altura de la planta (AP), biomasa fresca, índice de equivalencia de área (IEA), índice de selección ponderada (ISP), materia seca $(\mathrm{MS})$, proteína bruta $(\mathrm{PB})$, extracto etéreo $(\mathrm{EE})$, fibra detergente neutro (FDN), fibra detergente

\footnotetext{
* Autor para correspondencia. Correo electrónico: jeguevarao@unal.edu.co

1 Universidad Nacional de Agricultura, Facultad de Ciencias agropecuarias, Catacamas-Honduras. (D) 0000-0002-4727-491X.
}

Aceptado: 05/10/2020

\section{ABSTRACT}

\begin{abstract}
Selection of type IV climbing leguminous in association with corn for bovine feeding. Introduction. Ruminant production depends almost exclusively on forages that desirably have a high nutritional quality, so updated studies on this attribute, are required. For the present study, an analysis of the association of grasses and legumes as an alternative to increase the nutritional value of forages was carried out. Objective. To assess the agronomic and nutritional quality of maize associations with legumes of climbing habit IV for bovine feeding. Materials and methods. A ramdomized complete block design was used with 7 treatments and 3 replications consisting of: $\mathrm{T}_{1-6}=$ Corn + Blue lake, Beijin, Chi tou, Pritkar, Beans, CIAT 16806 and $\mathrm{T}_{7}=$ Corn ICAV305. Plant height $(\mathrm{PH})$, fresh biomass, area equivalence index (AEI), weighted selection index (WSI), dry matter (DM), crude protein (CP), ether extract (EE) , neutral detergent
\end{abstract}

2 Universidad Nacional de Agricultura, Facultad de Ciencias agropecuarias, Catacamas-Honduras. (iD) 0000-0002-4209-4629.

3 Universidad Nacional de Agricultura, Facultad de Medicina Veterinaria y Zootecnia, CatacamasHonduras.

(iD) $0000-0001-8031-8307$. 
ácida (FDA) y lignina (LDA). Los datos fueron analizados estadísticamente y sometidos al ISP e IEA. Resultados. Se incrementó la cantidad de biomasa fresca en asocios (promedio 72,2 \pm 5 ton. $\left.\mathrm{ha}^{-1}\right)$ respecto al monocultivo de maíz $(64,82$ ton.ha-1 ${ }^{-1}$. La concentración de MS se afectó ( $\mathrm{r}=$ $-0,37$ Pearson $<0,05$ ) por la cantidad de biomasa aportada por las leguminosas para una media de $18,4 \%$ en asocios y $22,40 \%$ del maíz. El contenido de PB mostró superioridad de 3-5 g/100g MS sobre el maíz. El contenido de FDN en asocio fue inferior (42,71\% Maíz + Lago azul) respecto al maíz (monocultivo 55,82\%). El IEA para el asocio Maíz + Lago azul presentó mayor eficiencia $(53,2 \%)$ frente a los monocultivos. El índice de selección ponderada estimado al considerar AP, Biomasa, PB y MS total mostró los tratamientos: Maíz + Lago azul y Beijín con la mejor posición jerárquica. Conclusión. De mayor a menor, asociar maíz ICA-V305 con Lago azul, Judías o Chi tou son mejor alternativa alimentaria para el ganadero (elaboración de ensilajes y/o alimento fresco de corte).

\section{INTRODUCCIÓN}

La producción animal se basa en la alimentación con forrajes. Por ende, es necesario contar con este recurso que sea de alto valor alimenticio, con fin de proporcionar los requerimientos nutricionales, de acuerdo con las necesidades de los animales (proteínas y carbohidratos) para lograr que estos expresen al máximo su potencial genético (Percy 2015). Los costos de alimentación en la producción lechera representan el renglón más alto dentro de la inversión total, ya que inciden en un 50\% del valor total del litro de leche (Hazard 2009). La calidad nutritiva de los forrajes incrementa los parámetros productivos de la ganadería, aunque con frecuencia no se tiene clara la idea de cómo mejorar este atributo. Actualmente se continúan estudios para fiber (NDF), acid detergent fiber (ADF), and lignin (LDA) were evaluated. The data were statistically analyzed and submitted to the WSI and AEI. Results. The amount of fresh biomass in association increased (average $72.2 \pm 5$ ton. $\mathrm{ha}^{-1}$ ) compared to maize monoculture (64.82 ton.ha $\left.{ }^{-1}\right)$. DM concentration was affected $(\mathrm{r}=$ -0.37 Pearson $<0.05$ ) by the amount of biomass provided by legumes for an average of $18.4 \%$ in association and $22.40 \%$ of corn. CP content showed superiority of 3-5 g / 100g DM over corn. NDF concentration was lower in associations $(42.71 \%$ Corn + Blue lake) compared to corn monoculture $(55.82 \%)$. AEI for of corn + blue lake association presented greater efficiency (53.2\%) compared to monocultures. The estimated weighted selection index considering $\mathrm{PH}$, biomass, $\mathrm{CP}$ and total $\mathrm{DM}$, showed the treatments: corn + Blue lake and Beijin with the best hierarchical position. Conclusion. From highest to lowest, associating ICA-V305 corn with Blue lake, Beans or Chi tou are a better feed alternative for the farmer (production of silages and / or fresh cut feed).

dar respuesta al asocio de gramíneas y leguminosas como alternativa para incrementar el valor nutricional de los forrajes.

La creciente demanda de proteínas para consumo humano y animal en los países en desarrollo, y los altos costos de importación justifican la exploración del uso de fuentes alternas de proteína como es el caso de las leguminosas, que por su adaptabilidad y contenido de proteína podría tener buen resultado asociada con gramíneas (Ríos 1993).

Las características nutritivas, de la mayoría de los alimentos clasificados como forrajes presentan alto contenido de fibra cruda y bajos contenidos relativos de proteína y de energía (Fassio et al. 2018). "Al hacer referencia a forrajes de alta calidad, es porque los mismos tienen moderados a elevados tenores proteicos (más del 
$12 \%)$ y bajos a moderados niveles de fibra ( $28 \%$ a $60 \%$ ), con más del $60 \%$ de digestibilidad" (Almeida 2014).

En ese sentido, la asociación de plantas de diversas especies tiene como finalidad optimizar los recursos, generar ventajas agronómicas, tales como el uso intensivo de la tierra, la diversificación de cultivos y de fuentes de alimento (Pérez et al. 2013). Pereira y Pacheco (2003) señalan que el asocio de gramíneas con leguminosas mejora la fertilidad del suelo, ya que se obtiene mayor producción de biomasa por área, mejor calidad y a la vez, se beneficia la persona productora porque con la cosecha del cultivo en algunos casos cubre en gran medida los costos de la renovación de la pradera y tiene disponibilidad de mejores forrajes. Por su parte Villaquirán y Lascano, citado por Rojas y Olivares (2005), demostraron que las leguminosas incrementan el valor nutritivo de la gramínea asociada, en lo que se refiere a los contenidos de proteína.

\section{Producción animal en pasturas asociadas}

Se ha comprobado que al utilizar asocios de gramíneas con leguminosas, hay aumentos de ganancia de peso (Gomide y Obeid 1979). Asimismo, CIAT (1979) muestra que la presencia de leguminosas ( $P$. phaseoloides y $S$. capitata) en forrajes de gramíneas mejoradas $(B$. decumbens y $A$. gayanus) aumentó la ganancia de peso vivo (40 y $60 \mathrm{~kg}$ ). Otras investigaciones citan que la ganancia de peso/animal ha sido siempre más elevada en los forrajes consorciados que en los forrajes exclusivos no abonados con nitrógeno. En los forrajes consorciados, Bryan y Evans (1973) observaron una correlación positiva significativa $(r=0,89)$ entre ganancia de peso vivo/animal y porcentaje de leguminosa en el forraje disponible.

\section{MATERIALES Y MÉTODOS}

Para la ejecución del experimento se utilizó maíz amarillo (Zea mays) variedad ICA
V-305, y 6 genotipos de leguminosas Phaseolus spp. de crecimiento indeterminado trepador, tipo IV: Lago azul, Beijín, Chi tou, Pritkar, Judías y CIAT-16806, colectado en el banco de germoplasma de frijol del Centro Internacional de Agricultura Tropical "CIAT" con la selección de los materiales utilizados se buscó la asociación de gramíneas con leguminosas de crecimiento indeterminado para aumentar el rendimiento y la calidad de la biomasa obtenida y con ello, la productividad de los hatos ganaderos (Lascano y Ávila 1991).

Siembra de maíz y leguminosas. Se utilizó una densidad de siembra en maíz de 62 500 plantas.ha $^{-1}$, bajo un marco de plantación de $0,80 \mathrm{~m}$ entre surco y $0,20 \mathrm{~m}$ entre planta, 20 días después de sembrado el maíz (ddsm) para 6 genotipos de leguminosas. La siembra de las leguminosas fue a $0,4 \mathrm{~m}$ entre plantas simples en el surco establecido previamente con maíz, para una densidad total de 32250 plantas.ha $^{-1}$ de leguminosa, lo que representa una relación de plantas/área del $65 \%$ del maíz y 35\% de leguminosas.

Fertilización. Se realizó una aplicación de urea diluida en agua [concentración al $2 \%$, 20 g..$^{-1}$ agua] a razón de 50 ml.planta ${ }^{-1}$ de maíz a los 10 días ddsm y una fertilización granular a los $25 \mathrm{ddsm}$, con una relación 2:1 de urea y fórmula 15-15-15 para una cantidad de la mezcla por área de $410 \mathrm{~kg} \cdot \mathrm{ha}^{-1}$. La fertilización de las leguminosas fue foliar aplicada a los 7, 14 y 21 días de establecido el cultivo y se utilizó la fórmula Desarrollo (30-20-7) a una concentración de $[0,8 \%]$.

Diseño experimental. Se utilizó un diseño de bloques completos al azar con 7 tratamientos (Tabla 1) y 3 repeticiones, para un total de 21 observaciones con un área útil de $8 \mathrm{~m}^{2}$ por observación. 
Tabla 1. Tratamientos: variedad de maíz (ICA V-305) evaluada en asociación con variedades de frijol de crecimiento indeterminado (tipo IV) establecidas bajo una relación de siembra 65:35.

\begin{tabular}{cl}
\hline No Tratamiento & \multicolumn{1}{c}{ Descripción } \\
\hline T1 & Maíz + Lago azul \\
T2 & Maíz + Beijín gun du \\
T3 & Maíz + Chi tou \\
T4 & Maíz + Ptitkar \\
T5 & Maíz + Judías \\
T6 & Maíz + CIAT 16806 \\
T7 & Maíz (Monocultivo) \\
\hline
\end{tabular}

Descripción de tratamientos. Se estudiaron 6 variedades de leguminosas en asocio con maíz, donde se emplearon 7 tratamientos: 6 asocios (maíz + leguminosas) y maíz en monocultivo, descritos en la Tabla 1.

\section{Variables evaluadas}

Altura de planta. Para realizar estas mediciones se tomaron en cuenta 2 consideraciones:

En el caso del maíz, el criterio de medición fue a los 80 ddsm; se midió la altura en centímetros sobre el eje principal, desde el punto de inserción de las raíces hasta la base de la espiga (CIAT 1993).

Para las leguminosas de hábito trepador tipo IV, se siguió la metodología descrita por CIAT (1993) que consiste en medir la altura (cm) desde la parte basal del tallo, hasta el último meristema apical. Esa medición se realizó con estadía, al final del ciclo del cultivo.

Índice de equivalencia de área (IEA). Se utilizó la fórmula de índice de equivalencia de área (IEA) propuesta por Vieira (1985), adaptada para biomasa que consiste en relacionar la producción de biomasa del cultivo en asocio y la del monocultivo, mediante la presente fórmula:

$$
\boldsymbol{I E A}=\frac{C A}{M A}+\frac{C B}{M B}
$$

Donde:
IEA = índice de equivalencia de área o de tierra.

$\mathrm{CA}, \mathrm{CB}=$ productividades de biomasa fresca (ton. ha ${ }^{-1}$ ) de cada cultivo (maíz y leguminosa) en asocio.

$\mathrm{MA}, \mathrm{MB}=$ productividades de biomasa de las especies evaluadas en monocultivo.

Los resultados obtenidos de la fórmula se compararon mediante la regla de decisión propuesta por Vieira (1985), donde el asocio será eficiente si el IEA es superior a uno y perjudicial al ser inferior a uno. Para garantizar la equivalencia del índice, los cultivares en monocultivo de las leguminosas fueron establecidas bajo un mismo esquema en cuanto al marco de siembra, riego, manejo técnico.

Producción de biomasa fresca. Se midió el rendimiento de biomasa fresca por área a los 80 ddsm (etapa fenológica maíz $=$ VT y R8 en leguminosas), según metodología descrita por Toledo y Shultze-Kraft (1982).

Características bromatológicas. Las características bromatológicas de los cultivares fueron analizadas con la metodología propuesta por Estrada y Leterme (2010) para los principios inmediatos determinados por el método de Weende y Van Soest.

\section{Método Weende}

Materia seca. Se estimó por el método gravimétrico propuesto por Alarcón (2019). Se sometió por triplicado una muestra inicial de 350 g. a $60^{\circ} \mathrm{C}$ por 72 horas, posteriormente se molió en molino de martillos a partículas menores de 1 mm. La muestra (2-5 g) nuevamente se sometió por triplicado a secado en estufa de aire forzado a $105^{\circ} \mathrm{C}$ por 12 horas.

Cenizas. Se analizó el contenido de ceniza por triplicado de la muestra al incinerar 3-5 gramos de la harina en un horno tipo mufla por 5 horas a una temperatura oscilante de $550^{\circ} \mathrm{C}-600^{\circ} \mathrm{C}$. 
Proteína bruta. Se determinó por el método de Kjendhal bajo la metodología Buchí, que consiste en la utilización de reactivos para la valoración cuantitativa de las proteínas a través de medidas colorimétricas.

Para la determinación del valor total de proteína se empleó la siguiente fórmula (Estrada y Leterme 2010):

$\%$ de Proteína $=\%$ de Nitrógeno X 6,25.

Extracto etéreo. Su determinación se fundamenta en la propiedad de los lípidos de ser solubles en disolventes orgánicos apolares como el éter. Es un método gravimétrico que consiste en el aislamiento de la grasa por disolución en el disolvente (éter de petróleo) con la ayuda del extractor Soxhlet.

Los componentes estructurales del alimento (FDN: fibra detergente neutro; FDA: fibra detergente ácido y LDA: lignina detergente ácido) se determinaron mediante los métodos Van Soest y ANKOM descritos por Estrada y Leterme (2010).

Análisis estadístico. Se realizó un análisis de varianza y prueba de media Duncan al $5 \%$ de probabilidad para las variables: altura de planta, producción de biomasa fresca, porcentaje de materia seca, proteína cruda, cenizas, EE, FDN, FDA, LDA. Los datos del uso equivalente de área y los de las leguminosas en monocultivo se analizaron con estadística descriptiva.

Se hizo un análisis de correlación lineal por el método de Pearson $(0,05)$ paras las variables evaluadas de los asocios de maíz con leguminosa. De acuerdo con Vinuesa (2016), esta medida o índice de correlación $r$ puede variar entre $-1 \mathrm{y}+1$, ambos extremos con indicaciones de correlaciones perfectas, negativa y positiva respectivamente. El modelo aditivo lineal empleado fue:

$$
\mathrm{Xij}=\mu+\mathrm{Bj}+\mathrm{Tij}+\mathrm{Eij}
$$

Donde:

$\mathrm{Xij}=$ variable aleatoria observable.

$\mu=$ media general.

$\mathrm{Bj}=$ efecto del $\mathrm{j}$-ésimo bloque.

$\mathrm{Ti}=$ efecto de la variedad de frijol en asocio.

Eij $=$ efecto del error experimental.

$\mathrm{i}=1 \mathrm{~T}$ (tratamiento).

$\mathrm{j}=1 \mathrm{r}$ (repeticiones).

Selección de leguminosas a través del índice de selección ponderada (ISP). Se utilizó el índice de selección ponderada descrito por Ortiz et al. (2013), con las variables de producción de biomasa, altura de leguminosa y el porcentaje de proteína y materia seca. El factor de ponderación utilizado se definió según el nivel de importancia a los resultados "criterio de las personas investigadoras". Los valores obtenidos del ISP, se posicionaron jerárquicamente de acuerdo con su valor en orden descendente para identificar los mejores en orden ascendente. El modelo matemático empleado responde a:

$$
I S P_{(K)}=\sum_{1}^{6} P i \frac{\left(\bar{X}_{i(K)}-\bar{X}_{g(i)}\right)}{S_{g(i)}}
$$

Donde:

$I S P_{(K)}=$ índice de selección ponderada o estandarizado para la introducción k-ésima (k: $1,2,3 \ldots)$.

$P i=$ factor de ponderación asignado al i-ésimo descriptor (i: 1, 2, 3...). Se distribuye entre las variables estudiadas. Es un valor a libre albedrio de la persona investigadora, según sea el valor práctico del carácter en estudio (su sumatoria debe ser igual a uno). 
$\overline{\mathrm{X}} i(K)=$ promedio del i-ésimo descriptor en la k-ésima introducción.

$\overline{\mathrm{X}} g(i)=$ promedio general de la colección para el i-ésimo descriptor.

$\operatorname{Sg}(K)=$ desviación estándar general del i-ésimo descriptor con las k-ésima introducciones.

\section{RESULTADOS}

Se evaluó el efecto de asociar maíz (variedad ICA V-305) con leguminosas de crecimiento indeterminado tipo IV, del cual se obtuvieron resultados positivos para cada una de las variables evaluadas. Los parámetros evaluados en la asociación de maíz con leguminosas fueron sometidas a un análisis de varianza $(\mathrm{p} \leq 0,05)$, con los siguientes resultados (Tabla 2).

Tabla 2. Parámetros productivos obtenidos de la asociación de maíz (ICA V-305) con leguminosas de hábito trepador tipo IV establecidas bajo una relación de siembra 65:35.

\begin{tabular}{|c|c|c|c|c|c|c|c|}
\hline \multirow{2}{*}{$\begin{array}{l}\text { Variable de } \\
\text { respuesta }\end{array}$} & \multicolumn{6}{|c|}{ Maíz en asocio con } & \multirow{2}{*}{ Maíz } \\
\hline & Lago azul & Beijín & Chi tou & Pritkar & Judías & $\begin{array}{l}\text { CIAT } \\
16806\end{array}$ & \\
\hline Altura de maíz (cm) & 229,07 & 238,77 & 235,97 & 234,00 & 232,4 & 230,03 & 234,27 \\
\hline Altura leguminosa ${ }^{* *}$ & $356,8 \mathrm{a}$ & $406,8 \mathrm{a}$ & 367,13 a & $131,1 \mathrm{~b}$ & $424,7 \mathrm{a}$ & $384,2 \mathrm{a}$ & - \\
\hline Biomasa t.ha ${ }^{-1^{*}}$ & 76,97 a & $72,22 \mathrm{ab}$ & $78,55 \mathrm{a}$ & $73,9 \mathrm{ab}$ & $73,5 \mathrm{ab}$ & $66,84 \mathrm{~b}$ & $64,8 \mathrm{~b}$ \\
\hline$\% \mathrm{MST}^{* *}$ & $17,77 \mathrm{c}$ & $16,44 \mathrm{c}$ & $17,43 \mathrm{c}$ & $21,8 \mathrm{ab}$ & $18,2 \mathrm{bc}$ & $18,7 \mathrm{bc}$ & $23,6 \mathrm{a}$ \\
\hline$\%$ Cenizas & 8,31 & 8,51 & 8,25 & 9,54 & 8,9 & 9,38 & 8,76 \\
\hline \% Proteína* & $17,69 \mathrm{a}$ & $17,11 \mathrm{a}$ & 16,65 a & $16,40 \mathrm{a}$ & $16,4 \mathrm{a}$ & $16,14 \mathrm{a}$ & $12,6 \mathrm{~b}$ \\
\hline$\% \mathrm{EE}^{* *}$ & $2,36 \mathrm{~d}$ & $4,11 \mathrm{a}$ & $3,92 \mathrm{ab}$ & $4,4 \mathrm{a}$ & $3,2 \mathrm{bc}$ & $3,15 \mathrm{c}$ & $2,9 \mathrm{~cd}$ \\
\hline$\% \mathrm{FDN}^{* *}$ & $42,71 \mathrm{~d}$ & $42,61 \mathrm{~d}$ & $49,91 \mathrm{~b}$ & $45,63 \mathrm{c}$ & $46,16 \mathrm{c}$ & $46,72 \mathrm{c}$ & $55,8 \mathrm{a}$ \\
\hline$\%$ FDA & 24,76 & 25,43 & 22,6 & 21,8 & 26,3 & 25,85 & 28,2 \\
\hline$\%$ LDA $^{*}$ & $4,60 \mathrm{c}$ & $4,62 \mathrm{c}$ & $6,92 \mathrm{a}$ & $4,53 \mathrm{c}$ & $6,1 \mathrm{abc}$ & $5,05 \mathrm{bc}$ & $6,6 \mathrm{ab}$ \\
\hline
\end{tabular}

Donde: *significancia estadística $(\mathrm{p} \leq 0,05), * *$ significancia estadística $(\mathrm{p} \leq 0,001)$.

Altura de la planta. La altura de la planta de maíz no se observó modificada por el sistema de cultivo. Probablemente se debió a que el maíz tuvo 20 días de establecido al sembrar las leguminosas y no se ejerció una considerable competencia por agua, luz y nutrientes. La altura de las leguminosas asociadas mostró diferencia entre las variedades $(\mathrm{p}<0,01)$, donde la variedad Pritkar presentó la menor altura $(131,1 \mathrm{~cm})$. Esta variable se modificó aparentemente en un $3 \%$ en asocios frente a los monocultivos. Por lo que el promedio general/genotipos en monocultivo fue de 3,63 y $3,45 \mathrm{~m}$ de altura en asocios.

Producción de biomasa fresca. La producción de biomasa fresca (ton.ha ${ }^{-1}$ ) del cultivo de maíz en asocio con (Phaseolus spp.) se determinó a los 80 ddsm (etapa fenológica maíz= VT y R8 en leguminosas). Se encontraron diferencias entre tratamientos $(p<0,05)$. El rendimiento promedio del forraje del maíz en monocultivo fue de 64,82 ton.ha ${ }^{-1}$ lo cual mejoró considerablemente 
el rendimiento al asociarlo con la variedad de frijol Lago azul (76,97 ton.ha $\left.{ }^{-1}\right)$ y Chi tou $(78,55$ ton.ha- $\left.{ }^{-1}\right)$. Con base en estos resultados en algunos casos aparentemente los asocios aportan al menos 8 toneladas más de lo que puede producir el maíz sembrado en monocultivo, excepto si se asocia con la variedad de frijol CIAT 16806, que el aumento aparente fue de 2 toneladas de alimento. La importancia de generar mayor biomasa por área indica eficiencia en el uso de la tierra, con la optimización de la carga animal.

La producción de biomasa fresca (ton.ha ${ }^{-1}$ ) de las leguminosas bajo 2 sistemas de siembra (asocio: $4,74 \pm 2,13$ y monocultivo: $20,21 \pm 7,48)$ fue afectado al ser cultivado con maíz $(75 \pm 3 \%$ de reducción del rendimiento) en un arreglo de siembra 65:35.

Porcentaje de materia seca total. Los indicadores bromatológicos mostraron que la cantidad de materia seca se afectó por el tipo de tratamiento $(\mathrm{p} \leq 0,05)$, al reducir la concentración en los asocios, por lo que el maíz en monocultivo mostró los mayores valores $(23,64 \%)$ comparables únicamente al asociarlo con la variedad de frijol Beijín (21,8\%).

Mediante el análisis de correlación lineal Pearson, se comprobó que existe una relación negativa $(r=-0,37)$ entre la biomasa fresca y $\%$ MST donde se asume que la biomasa aportada por las leguminosas estimula negativamente el contenido de materia seca.

Porcentaje de cenizas. La concentración de cenizas no se afectó por el tipo de tratamiento $(\mathrm{p} \leq 0,05)$, al mostrar valores que oscilaron entre 8,25 a $9,54 \%$ en asocios; $10,35 \pm 1,70 \%$ para las leguminosas en monocultivo y $8,46 \%$ en maíz. Se evidenció una correlación negativa $(r=-0,41$; Pearson 0,05 ) entre la producción de biomasa y el contenido de cenizas; a mayor biomasa en los asocios, mayor aporte de las leguminosas a esa característica, lo que representa que estas hacen sensible el contenido de minerales.

Porcentaje de proteína. El contenido de proteína con base en materia seca se incrementó al asociar maíz con las leguminosas evaluadas
( $p<0,05$; Tabla 2), con respecto al contenido en maíz sembrado en monocultivo (12,65\% PB) y menor en relación con el valor promedio obtenido para las leguminosas en monocultivo (19,13\%). El coeficiente de correlación entre biomasa fresca y proteína muestra que están ligadas positivamente $r=0,681$. El aporte de las leguminosas a la biomasa total estimuló positivamente el contenido de proteína de la mezcla a razón de 3 a 5 g/100 g de MS.

Porcetaje de extracto etéreo (EE). El contenido de grasas se afectó por el material de leguminosa utilizado, ya que se incrementó la concentración ( $\mathrm{g} / 100 \mathrm{~g}$ de MS) a partir del asocio de maíz con la variedad de frijol Pritkar $(4,44 \%)$ y Beijín $(4,11 \%)$, y lo redujo aparentemente en asocio con el frijol Lago azul $(2,36)$.

\section{Porcentaje fibra detergente neutro} (FDN). La concentración de FDN fue alterada por el tipo de tratamiento $(\mathrm{p}<0,05)$, ya que redujo su contenido al asociar el maíz con las variedades de frijol (tipo IV) evaluadas. Así la relación con mayor concentración después del maíz $(55,8 \%)$ fue con la variedad Chi tou (49,91\%). La contribución celular de las leguminas se evidenció por mayor rigidez que las gramíneas, aspecto que podría afectar la dilución de la FDN al asociarse.

Fibra detergente ácida y lignina (FDA Y LDA). La concentración de FDA no fue modificada por el tipo de tratamiento evaluado, ya que mostró concentraciones que oscilaron entre 21,8 a $28,2 \%$. En cambio, la concentración LDA se alteró por el tipo de tratamiento $(\mathrm{p}<0,05)$, ya que mostró reducciones aparentes al asociar el maíz con la variedad de frijol CIAT 16806 (5,05\%) y Judías (6,02\%), reducciones que alcanzaron diferencia estadística con el asocio de la variedad de frijol Beijín (4,62\%), Lago azul (4,60\%) y Pritkar (4,53\%).

Índice de equivalencia de área (IEA). Se estableció el IEA para el asocio de la variedad de maíz (ICA V-305) con 6 especies de frijol de crecimiento indeterminado Tipo IV, los resultados obtenidos muestran un índice de 1,112 a 
1,532 (Figura 1). El asocio de maíz con Chi tou fue el que presentó mejor aprovechamiento del suelo $(1,532)$, es decir que la variedad de maíz ICA V-305 requiere un 53,2\% más de área para producir la misma cantidad de biomasa que el asocio. Lo que supone una superioridad en comparación de los monocultivos de acuerdo con Vieira (1985) quien propuso que un asocio, sería rentable si su IEA expuesto, fuera superior a uno.

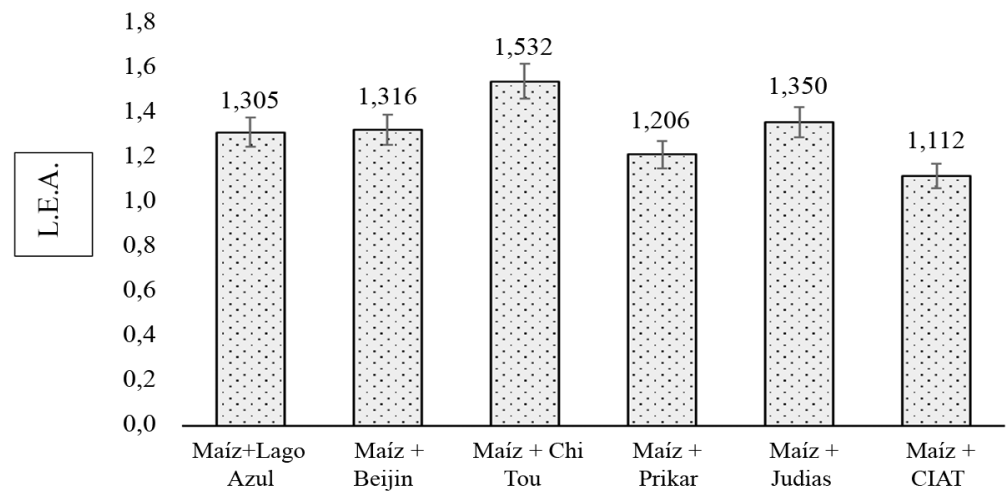

Figura 1. Índice de equivalencia de área del asocio de maíz (variedad ICA V-305) con 6 variedades de frijol establecidas bajo una relación de siembra 65:35.

Selección de leguminosa según el índice de selección ponderada (ISP). El ISP aplicado a los asocios de frijol con maíz junto con la altura de la leguminosa, producción de biomasa fresca, materia seca total y la proteína bruta mostró que el asocio de maíz con la variedad Lago azul y la variedad Judías fueron las que mostraron los mejores comportamientos productivos (Tabla 3 ) y que pueden ser de interés para la elaboración de ensilajes.

Tabla 3. Índice de selección ponderada del asocio de la variedad de maíz ICA V-305 con 6 genotipos de frijol (crecimiento tipo IV) establecidos bajo una relación de siembra 65:35.

\begin{tabular}{|c|c|c|c|c|c|c|}
\hline Asocio & ATL & $\mathrm{PBF}$ & $\%$ MST & $\% \mathrm{~PB}$ & $I S P$ & Ranking \\
\hline Maíz + Lago azul & 356,8 & 77,0 & 17,8 & 17,7 & 0,942 & 1 \\
\hline Maíz + Beijín & 406,8 & 72,2 & 16,4 & 16,4 & $-0,419$ & 5 \\
\hline Maíz + Chi tou & 367,1 & 78,6 & 17,4 & 16,4 & $-0,045$ & 3 \\
\hline Maíz + Pritkar & 131,1 & 73,9 & 21,8 & 16,6 & $-0,060$ & 4 \\
\hline Maíz + Judías & 424,7 & 73,5 & 18,3 & 17,1 & 0,398 & 2 \\
\hline Maíz + CIAT 16806 & 384,2 & 66,8 & 18,7 & 16,1 & $-0,816$ & 6 \\
\hline Promedio & 345,13 & 73,66 & 18,41 & 16,73 & & \\
\hline Desviación estándar & 107,78 & 3,87 & 1,85 & 0,57 & & \\
\hline CV $(\%)$ & 31,23 & 5,26 & 10,06 & 3,41 & & \\
\hline Factor de ponderación & 0,15 & 0,25 & 0,15 & 0,45 & & \\
\hline
\end{tabular}

ATL: altura total de leguminosas, PBF: producción de biomasa fresca, \%MST: porcentaje de materia seca total, $\% \mathrm{~PB}$ : porcentaje de proteína bruta. 


\section{DISCUSIÓN}

Altura de planta. La altura del cultivo de maíz no se modificó por la asociación con la leguminosa, lo que probablemente se debió a que el maíz tenía 20 días de establecido al sembrar las leguminosas y no se ejerció una considerable competencia por agua, luz y nutrientes. Darío et al. (2007), estimaron que el maíz asociado con leguminosas solamente se ve afectado en un 10 a $21 \%$ en su eco-fisiología, mientras que Titterton y Maasdorp (1997) indicaron que, si se siembra Dolichos lablab y Mucuna deeringiana 2 semanas después de la siembra del maíz, el rendimiento del maíz no muestra cambios y que el rendimiento de MS de la leguminosa representaba cerca de $30 \%$ del total de la MS y como resultado, un ensilaje con un contenido de proteína bruta próximo al $10,5 \%$.

Biomasa. El rendimiento en biomasa mejoró al asociar maíz con leguminosas (promedio de 66,8 a 78,5 toneladas.ha ${ }^{-1}$ ) respecto a maíz en monocultivo (64,8 toneladas), producción que se encuentran entre el rango de valores reportados por Bernal (1991). Para el maíz forrajero manejado en sistema de riego (60 a 80 ton.ha ${ }^{-1}$ cosechado a los 90 días). Castillo et al. (2009) se identificaron valores superiores que oscilan entre 70 y 92 ton. ha $^{-1}$ cortados a los 85 días, en la zona de Durango, México.

Materia seca. Valores similares a los obtenidos (23,6\% MS para maíz en monocultivo y 16,4 a $21,8 \%$ MS en asocios) fueron reportados por Arteta y Zamora (2005) pues se registró un $22,40 \%$ de MST para el maíz en monocultivo y $18,4 \%$ en la mezcla del maíz con las leguminosas. Así también, Pérez et al. (2013) concluyen que al asociar maíz con frijol disminuye la concentración de materia seca.

Cenizas. La concentración de cenizas no se alteró por el tipo de tratamiento, ya que presentó valores que oscilaron entre 8,25 a $9,54 \%$. Valores inferiores $(5,8$ a $7,5 \%)$ fueron reportados por Castillo et al. (2009) al evaluar la asociación de maíz con $V$. radiata.

Proteína. La concentración de proteína mejoró al asociar el maíz con las leguminosas evaluadas. El promedio de PB alcanzado en este ensayo fue de $16,73 \%$. Resultados que corroboran los supuestos, que la manera de aumentar el contenido de proteína en los forrajes es por medio de la asociación de gramíneas con leguminosas, ya que debido a su gran valor proteico son capaces de provocar aumentos de proteína por unidad de área para generar material de alto valor nutricional. El porcentaje de proteína bruta en leguminosas fue afectado por distintos factores: especie, estado fenológico, parte de la planta, nivel de fertilidad del suelo. Su tendencia fue decreciente hacia la madurez con rangos entre 10 y $30 \%$ (Parsi et al. 2002). El promedio de PB alcanzado en este ensayo fue superior al obtenido por Jiménez et al. (2005) en ensilado de Canavalia ensiformes (leguminosa) en asocio con maíz $(8,7 \%$ PB). El aumento de proteína en los asocios concuerda con lo reportado por Castillo et al. (2009), en donde estudiaron el valor nutricional de maíz asociado con Vigna radiata bajo una relación de siembra de 70:30.

Extracto etéreo. La concentración de extracto etéreo del alimento varió ligeramente por el tipo de leguminosa asociada con maíz (asocio: 2,36 a 4,4\% EE y maíz monocultivo: $2,9 \%)$. Los valores medios obtenidos estuvieron entre datos ya reportados para leguminosas (2\%) y gramíneas (3\%) (Parsi et al. 2002) aunque superiores a los reportados por Castillo et al. (2009) en una asociación de maíz con vigna (Vigna radiata) [1,02 a 1,69\%].

Fibra detergente neutro (FDN). La concentración de FDN se redujo al asociar el maíz en un $55,8 \%$, con las variedades de frijol evaluadas con rango en asocio de 42,71 a 49,91\%. Esos resultados están en las categorías evaluadas por Castillo et al. (2009), respecto al contenido nutricional del maíz asociado con $V$. radiata lo que 
le representó un $53,38 \pm 7,75 \%$ en base a materia seca. La ligera reducción probablemente se deba a la contribución celular de las leguminas, que por lo general son más rígidas que las gramíneas, aspecto que podría afectar la FDN del alimento al modificar la celulosa, hemicelulosa, lignina, cutina y sílica. Las diferencias encontradas entre monocultivo respecto a lo identificado por otros autores (Rojas y Olivares 2005) podría deberse a las condiciones de manejo, como el momento de corte, variedad y clima, entre otros, a los que haya sido sometido el cultivo.

Fibra detergente ácida y lignina (FDA Y LDA). Los valores medios de FDA no se modificaron por el tipo de material genético asociado, sin embargo, al asociar el maíz (28,2\% FDA) con las leguminosas, la concentración se redujo entre un 26,3 y un $21,8 \%$. Los valores obtenidos fueron inferiores a los reportados $(30,5 \%)$ por Castillo et al. (2009) aunque se encuentran entre el 24 al 26\%, rango identificado para el maíz por Demanet (2017). Es posible además, que al obtener menores concentraciones de FDA en asocio, favorecerá la digestibilidad total del forraje y por el contrario, cuanto más alta, será menos digestible (Gallardo 2007).

En asocios de maíz con leguminosas, el contenido de lignina aumenta debido a que las leguminosas por su naturaleza contienen más lignina que las gramíneas (Bash y Calsamiglia 2006), sin embargo, este hecho se presentó únicamente al asociar el maíz con la variedad de frijol Chi tou. No obstante, los valores obtenidos en los asocios (5,72 $\pm 2 \%$ LDA) fueron superiores a los reportados por Gallardo (2007) al cultivar maíz con canavalia (2,8 a 3,6\% LDA) y dentro del rango (3 a 5\%) reportado por Castillo et al. (2009) aunque menores al obtenido en este estudio al cultivar el maíz en monocultivo $(6,6 \%)$. El maíz en monocultivo para ensilado ha sido referencia para los ganaderos, si se considera que LDA actua como una barrera para la digestión microbiana ruminal de la celulosa y la hemicelulosa (Gallardo 2007), el material (Lago azul,
Beijin y Pritkar) de los cultivos asociados con maíz podrían presentar menor resistencia a la digestibilidad microbiana.

Índice de equivalencia de área e índice de selección ponderada. Los asocios de maíz con frijol (relación de siembra de 65:35) favorecieron un mejor aprovechamiento del área de cultivo (1,112-1,532), donde el mayor IEA correspondió al asocio de la variedad de maíz (ICA V-305) con la variedad de frijol Chi tou. Los resultados obtenidos fueron consistentes también con los reportes de Tirabassi et al. 2012, Lapas 2014 y Diehl 2015, quienes identificaron el asocio como alternativa para mejorar rendimientos de pequeños y medianos productores, ya que los sistemas combinados permiten mayor aprovechamiento por área.

El ISP se determinó por medio de factores relacionados a cantidad y calidad del forraje (altura de leguminosa, producción de biomasa fresca, materia seca total y proteína bruta), lo que permitió identificar el asocio de maíz con frijol variedad Lago azul (ISP $=0,942 ; 76,97$ ton.ha ${ }^{-1}$ ) como mejor alternativa para el ganadero (elaboración de ensilajes y/o alimento fresco de corte), y al asocio de maíz con Judías (ISP 0,398; 73,5 ton.ha $\left.{ }^{-1}\right)$ y maíz con Chi tou $(-0,045)$ también como alternativas. Este último además presenta el mayor rendimiento en biomasa fresca $(78,55$ ton.ha' ${ }^{-1}$ ) y el mejor IEA $(1,523)$.

\section{CONCLUSIONES}

Se determinó que la proteína bruta del alimento para el animal se incrementó en valores de 3 a $5 \%$ de MS al asociar el maíz ICA V-305 con variedades de frijol de hábito trepador tipo IV (Lago azul, Beijín, Chi tou, Pritkar, Judías y CIAT 16806) en una relación de siembra de 65:35.

La producción de biomasa fresca se incrementó al asociar la variedad de maíz ICA V-305 (promedio 64,8 ton.ha ${ }^{-1}$ en monocultivo) con 
leguminosas. Alcanzó significancia en asocio con la leguminosa Lago azul, ya que representó 12,17 ton. ha ${ }^{-1}$ sobre el promedio y también con Chi tou que mostró 13,75 ton.ha ${ }^{-1}$ sobre el promedio y entre 7,4 a 9,1 ton. ha ${ }^{-1}$ sobre el promedio en asocio con la variedad Beijín, Judías y Pritkar.

El asocio de maíz con variedades de frijol afectó la concentración de FDN, FDA y LDA, ya que la concentración se redujo en algunas pruebas, de forma significativa o aparente. Las reducciones en FDA y LDA podrían ser beneficiosas al incrementar la digestibilidad total del forraje para rumiantes si se da menor resistencia a la acción enzimática bacteriana, sin embargo, aún se requiere de más valoraciones.

El índice de equivalencia de área fue superior a uno al asociar la variedad de maíz ICA V-305 con las variedades de frijol (IEA 1,022 a 1,532), en asocio con la leguminosa Chi tou que además evidenció mayor eficiencia en el uso del terreno al haber establecido una relación de siembra de 65:35.

El índice de selección ponderada se calculó a partir de la altura de la leguminosa, biomasa fresca por hectárea, y la concentración de materia seca y proteína, donde el asocio de maíz con la variedad de frijol Lago azul mostró el mayor índice, lo que favorece la nutrición del animal, ya que obtendría alimento en mayor cantidad y calidad.

\section{AGRADECIMIENTOS}

Al M.Sc. Gustavo López, Alexis Vallecillo y Ph.D. Sanín Ortiz y Luz Stella por su apoyo logístico durante la ejecución de la investigación en campo y laboratorio.

\section{LITERATURA CITADA}

Alarcón García, MÁ; López Vargas, JH; Restrepo Molina, DA. 2019. Caracterización de la funcionalidad tecnológica de una fuente rica en fibra dietaria obtenida a partir de cáscara de plátano / characterization of technological functionality of dietary fiber rich source obtained from plantain peel. Universidad Nacional de Colombia - Sede Medellín. Consultado 05 abr. 2019. Disponible en http://bdigital.unal.edu.co/37656/

Almeida, RM. 2014. Forrajes de calidad (en línea). Consultado 08 mar. 2019. Disponible en https://www.forratec. com.ar/manuales/pdfs/49-20150108122530-pdfEs.pdf

Arteta, D; Zamora, W. 2005. Efecto de dos tipos de asociaciones de maíz con cuatro leguminosas sobre la calidad del ensilaje. Tesis de Ingeniero Agrónomo. Tegucigalpa, Honduras, Escuela Agrícola Panamericana, Zamorano. 15 p.

Bash, A; Calsamiglia, S. 2006. La fibra en los rumiantes: ¿Química o física? (en línea). XXII Curso especialización FEDNA. Barcelona, España. p. 99-112. Consultado 08 mar. 2019. Disponible en http://produccion-animal.com.ar/informacion tecnica/manejo_del_alimento/100-fibra_en rumiantes.pdf

Bernal, J. 1991. Pastos y forrajes tropicales, producción y manejo. Bogotá: Banco Ganadero. Bogotá, Colombia. 544 p.

Bryan, WW; Evans, TR. 1973. Efectos de los suelos, los fertilizantes y las tasas de almacenamiento en los pastos y la producción de carne en el Wallum del sureste de Queensland. 1. Composición botánica y efectos químicos en plantas y suelos (en línea). Australian Journal of Experimental Agriculture 13(1):516-529. Consultado 08 mar. 2019. Disponible en http://www.publish.csiro.au/an/EA9730516

Castillo, MJ; Rojas, AB; Jones, W. 2009. Valor nutricional del ensilaje de maíz cultivado en asocio con vigna (Vigna radiata). Agronomía Costarricense 33(1):133146. Consultado 04 feb. 2019. Disponible en https:// www.researchgate.net/publication/28317065

CIAT (Centro Internacional de Agricultura Tropical, CO). 1979. Informe Anual del Programa de Frijol (en línea). Consultado 05 ago. 2018. Disponible en http://ciat-library.ciat.cgiar.org/Articulos Ciat/2015/14304.pdf

CIAT (Centro Internacional de Agricultura Tropical, CO). 1993. Descriptores varietales: Arroz, frijol, maíz, sorgo (en línea). Muñoz, G; Giraldo, G; Soto, JF. Cali, Colombia, Publicación $\mathrm{N}^{\circ}$ 177. Consultado 10 Feb. 2019. Disponible en https://cgspace.cgiar.org/ handle/10568/54651

Darío, L; Vargas, V; Clavijo, J. 2007. Relaciones de competencia entre el frijol trepador (Phaseolus vulgaris L.) y el maíz (Zea mays L.) sembrados en asocio (en línea). Universidad Nacional de Colombia. Consultado 06 ago. 2018. Disponible en https://revistas.unal.edu.co/index.php/refame/ article/view/29366/37146

Demanet, FP. 2017. Ensilaje de maíz, tiempo entre sellado y apertura- plan lechero watt's (en línea). Santiago, 
Chile. Consultado 10 Feb. 2019. Disponible en https://www.engormix.com/ganaderia-leche/ articulos/ensilaje-maiz-tiempo-entre-t41230.htm

Diehl, MS. 2015. Pastagens de capim elefante e azevém consorciadas com leguminosas (en línea). Tesis Ph.D. Brasil, Universidad Federal De Santa María. Consultado 06 ago. 2018. Disponible en https:// repositorio.ufsm.br/handle/1/4367

Estrada, F; Leterme, P. 2010. Análisis de alimentos y forrajes protocolo de laboratorios, Laboratorio de nutrición animal. Universidad Nacional de Colombia, Palmira, Colombia. p. 60

Fassio, A; Ibáñez, W; Fernández, E; Cozzolino, D; Pérez, O; Restaino, E; Pascal, A; Rabaza, C; Vergara, G. 2018. El cultivo de maíz para la producción de forraje y grano y la influencia del agua. INIA-Uruguay. Serie Técnica No 239 (en línea). Consultado 10 ene. 2019. Disponible en http://www.ainfo.inia.uy/ digital/bitstream/item/8897/1/st-239-2018.pdf

Gallardo, M. 2007. Dietas balanceadas con forrajes conservados: la importancia de diagnosticar la calidad nutricional (en línea). Consultado 04 ago. 2018. Disponible en http://www.produccion-animal. com.ar/informacion_tecnica/suplementacion/74dietas_balanceadas_forrajes_conservados.pdf

Gomide, JA; Obeid, LA. 1979. Fatores morfofisiológicos da rebrota do capimcolonião (Panicum maximum) (en línea). Rev. Soc. Bras. Zoot. 8(4):532-562. Consultado 03 ago. 2018. Disponible en https:// www.passeidireto.com/arquivo/48074434/ aspectosrecuperacaogramineasforrageiras $/ 4$

Hazard, S. 2009. Alimentación de Vacas Lecheras (en línea). INIA (Instituto de Investigaciones Agropecuarias, CL). Consultado 7 feb. 2010. Disponible en https:// infolactea.com/wp-content/uploads/2017/02/AD3.pdf

Lapas, H. 2014. Efecto de cultivo asociado de maíz (Zea mays L.) con arveja (Písum sativum L.) en el uso eficiente de la tierra, en condiciones de Pomacocha, Perú (en línea). Tesis Ing. Agrónomo. Universidad Nacional De Hu Anca Velica. Consultado 08 ene. 2019 Disponible en http://repositorio.unh.edu.pe/ bitstream/handle/UNH/174/TP\%20-\%20UNH\%20 AGRON.\%200055.pdf?sequence=1\&isAllowed=y

Lascano, CE; Avila, P. 1991. Potencial de producción de leche en pasturas solas y asociadas con leguminosas adaptadas a suelos ácidos (en línea). Pasturas Tropicales 13(3):2-10. Consultado 08 ene. 2019 Disponible en http://ciat-library.ciat.cgiar. org/pasturas_tropicales/Documents/1991-vol13rev1-2-3/Vol13_rev3_a\%F1o91_art2.pdf

Ortiz, GS; Vallejo, CF; Baena, GD; Estrada, SE; Valdés, RM. 2013. Zapallo para consumo en fresco y fines agroindustriales investigación y desarrollo. Colombia, Universidad Nacional de Colombia. 112 p.

Parsi, J; Godio, L; Miazzo, R; Maffioli, R; Echevarría, A y Provensal, P. 2002. Valoración nutritiva de los alimentos y formulación de dietas (en línea). Cursos de Producción Animal, FAV UNRC. Consultado 19 mar. 2019. Disponible en www.researchgate.net/ publication $/ 237311848$

Percy, C. 2015. Cultivo de pastos. Manual de Pastos Cultivados (en línea). (Swisscontact, IT). $40 \mathrm{p}$. Consultado 05 ago. 2018. Disponible en https:// www.swisscontact.org/fileadmin/user_upload/ COUNTRIES/Peru/Documents/Publications/ MANUAL_PASTOS_CULTIVADOS.pdf

Pereira, OI; Pacheco. YL. 2003. Implantaçao e conducto do Sistema Barreirao. In Kluthcouski, J; Stone, LF; Aidar, H (eds.). Integraçao lavoura-pecuaria. Embrapa, Arroz e feijao. San Antonio de Goias, Brasil (en línea). Consultado 03 ago. 2018. Disponible en http://www.scielo.org.co/scielo. php?script $=$ sci_nlinks\&ref $=000106 \&$ pid $=$ S0120 9965200800020001700014\&lng=en

Pérez, A; Martínez, E; Vélez, L; Cotes, J. 2013. Acumulación y Distribución de Fitomasa en el Asocio de Maíz (Zea mays L.) y Frijol (Phaseolus vulgaris L). Revista Facultad Nacional de Agronomía Medellín, Colombia (en línea). Consultado 07 ago. 2018. Disponible en http://www.scielo.org.co/pdf/rfnam/ v66n1/v66n1a04.pdf

Ríos, J. 1993. Uso de la Canavalia ensiformis en la alimentación de los rumiantes. Canavalia ensiformis (L.) DC Producción, procesamiento y utilización en la alimentación animal. Compilación de trabajos presentados en el primer Seminario-Taller sobre Canavalia ensiformis. Vargas, RE; Leon, A; Escobar, A (eds.). Fundación Polar, Venezuela. p. 241-250.

Rojas, S; Olivares, J. 2005. Manejo de praderas asociadas de gramíneas y leguminosas para pastoreo en el trópico (en línea). Tesis Ing. Agr. Consultado 06 ago. 2018. Disponible en https://www.researchgate. net/publication/237597794_Manejo_de_praderas _ asoci adas_de_gramineas_y_leguminosas_para_ pastoreo_en_el_tropico_Handling_of_prairies associated_of_gramineas_and_leguminosas_for_ pasturing_in_the_tropic

Tirabassi, LH; Soratto, RP; Matoso, AO; Abrahão, RC. 2012. Índice de equivalência de área do consórcio de milho e feijão-caupi (en línea). XXIV Congresso de Iniciação Científica da Unesp. Butucatu. Consultado 08 ene. 2019. Disponible en http://prope.unesp.br/ 
cic/admin/ver_resumo.php?area $=100073 \&$ subarea $=$ $22158 \&$ congresso $=34 \& \mathrm{CPF}=38646184870$

Titterton, M; Maasdorp, BV. 1997. Nutritional improvement of maize silage for dairying: mixed crop silages from sole and intercropped legumes and a long season variety of maize. 2. Ensilage (en línea). Journal of Food Science and Technology. 69:263270. Consultado 13 ene. 2019. Disponible en http:// www.fao.org/3/X8486S/x8486s06.htm
Toledo, JM; Shultze-Kraft. 1982. Metodología para la evaluación agronómica de pastos tropicales. CIAT, Cali, Colombia, p.110.

Vieira, CO. 1985. Feíjéo na cultivos consorciados. Universidad Federal de Vicosa. U.F. V. Brasil $134 \mathrm{p}$

Vinuesa, P. 2016. Correlación: teoría y práctica, CCGUNAM. México (en línea). Consultado 04 ago. 2018. Disponible en http://www.ccg.unam.mx/ vinuesa/ R4biosciences/docs/Tema8_correlacion.pdf 
